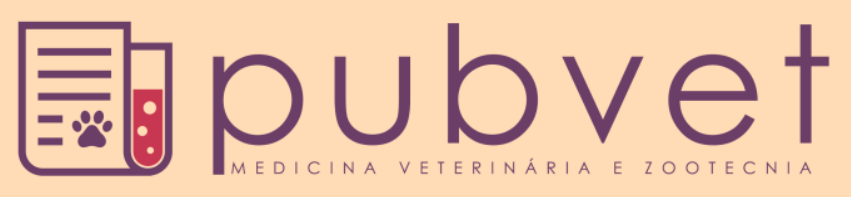

https://doi.org/10.22256/pubvet.v12n3a53.1-5

\title{
Abordagem cirúrgica do prolapso retal em felino: Relato de caso
}

\author{
Tatianne Alexandre Azevedo Viliotti ${ }^{\ominus}{ }^{*}$, Anne Nelizia Holanda de Lima ${ }^{2}$, Ingrid Rabelo \\ Rodrigues ${ }^{1}$, Aline Silveira Feitosa ${ }^{1}$, Rebeca de Melo Santos ${ }^{1}$, Sarah Ary Ceni', \\ Maria Ester Crispim Nogueira Fernandes $\bullet 1$, Richard Elaino de Oliveira Ferraz ${ }^{\ominus}$
}

${ }^{\text {I}}$ Graduação em Medicina Veterinária pela Faculdade Terra Nordeste (FATENE), Campus Caucaia, Ceará, Brasil.

${ }^{2}$ Medica Veterinária. Caucaia-CE, Brasil.E-mail: annenelizia@gmail.com

${ }^{3}$ Médico Veterinário. Professor da FATENE. Setor de cirurgia de pequenos animais. Campus Caucaia, Ceará, Brasil. E-mail: richard.ferraz@fatene.edu.br

*Autor para correspondência, E-mail: tatianneviliotti@gmail.com

RESUMO. O prolapso retal é uma protrusão ou eversão da mucosa retal sendo mais relatada em animais jovens, geralmente sendo uma consequência de distúrbios subjacentes que causam esforço intestinal e persistente, dentre outras causas. O objetivo do presente trabalho foi relatar o caso de um felino sem raça definida, com 2 meses de idade, atendido no Hospital Veterinário Metropolitano, Caucaia, Ceará, Brasil, apresentando anorexia, prostração e prolapso do reto com necrose. Foram realizados exames laboratoriais para avaliar funções biológicas do paciente. O tratamento cirúrgico eleito consistiu na aplicação de âncoras transversais e ressecção da massa prolapsada e aplicação de suturas únicas separadas para fazer a anastomose. O paciente foi acompanhado nas semanas seguintes e mostrou um retorno às funções fisiológicas normais da defecação, sem complicações de incontinência fecal. Conclui-se que a amputação retal pode ser uma alternativa para o tratamento cirúrgico do prolapso retal em felinos.

Palavras chave: Canal anal, intestinal, gato, protrusão

\section{Surgical approach of rectal prolapse in feline: Case report}

ABSTRACT. Rectal prolapse is a protrusion or eversion of the rectal mucosa from the anus and it is more reported in young animals. It is usually a consequence of underlying disorders intestinal diseases that cause diarrhoea and. The present study reports a case of rectal prolapsed in a cat puppy associated to necrose of the rectum, disquesia and emaciation. A feline S.R.D, 2 months old, was attended at the Metropolitan Veterinary Hospital, Caucaia, Ceará, Brazil, presenting the prolapsed rectum, tenesmus, dyskinesia, anorexia, emaciation, prostration and regions of necrosis in the prolapsed rectum. Haematological and biochemical tests revealed anemia and ALT increases. The surgical treatment consisted resection of restum and it was made an anastomose throue in the application of cross anchors and resection of the prolapsed mass and in the application of separate single sutures to make the anastomosis. The patient was reassessed in the following weeks and showed a return to the normal physiological functions of defecation. It is concluded that rectal amputation may be an alternative for the surgical treatment of rectal prolapse in felines.

Keywords: Anal canal, intestinal, cat, protrusion

\section{Enfoque quirúrgico del prolapso rectal en felino: Reporte de un caso}

RESUMEN. El prolapso rectal es una protrusión o eversión de la mucosa rectal siendo más reportada en animales jóvenes, generalmente siendo una consecuencia de disturbios 
subyacentes que causan esfuerzo intestinal y persistente, entre otras causas. El objetivo del presente trabajo fue reportar el caso de un felino mestizo, con 2 meses de edad, atendido en el Hospital Veterinario Metropolitano, Caucaia, Ceará, Brasil, presentando anorexia, postración y prolapso del recto con necrosis. Se realizaron exámenes de laboratorio para evaluar las funciones biológicas del paciente. El tratamiento quirúrgico elegido consistió en la aplicación de anclas transversales y resección de la masa prolapsada y aplicación de suturas únicas separadas para hacer la anastomosis. El paciente fue acompañado en las semanas siguientes y mostró un retorno a las funciones fisiológicas normales de la defecación, sin complicaciones de incontinencia fecal. Se concluye que la amputación rectal puede ser una alternativa para el tratamiento quirúrgico del prolapso rectal en felinos.

Palabras clave: Canal anal, intestinal, gato, protrusión

\section{Introdução}

O prolapso retal é a inversão de uma ou mais camadas do reto pelo ânus (Cunha et al., 2015) e é uma condição que pode ocorrer em qualquer idade ou espécie, porém mais de $80 \%$ são relatados em filhotes no primeiro ano de idade (Hedlund, 2008). Além disso, a taxa de ocorrência em animais do sexo masculino em comparação com os animais do sexo feminino são duas vezes maiores ( al., 2016). A depender das estruturas envolvidas o prolapso pode ser classificado como completo, onde todas as camadas retais são protrudidas ou incompleto, onde apenas a mucosa retal é prolapsada (Raut et al., 2008). Sua etiologia está comumente relacionada às causas digestivas, sendo observado geralmente em animais com diarreia, tenesmo graves (Cunha et al., 2015), endoparasitismo, doenças anorretais, que produzem constipação e disquesia, ou ainda, doenças do trato urinário inferior que causam estrangúria e disúria. A sintomatologia é caracterizada pela própria visualização da massa retal em forma de tubo, avermelhada ou enegrecida (casos crônicos), dores abdominais ao toque ou palpação, agressividade, inapetência, presença de sangramento ou não, constipação, diarreia, tendência de o animal lamber a massa prolapsada e a região perianal (Niebauer, 1993).

$\mathrm{O}$ tratamento dependerá do grau, cronicidade e recidivância do prolapso. Quando agudo tem tratamento fácil e conservativo, no entanto quando crônico pode necessitar de ressecção. Se a doença está na fase aguda, com lesão tecidual e edema mínimo, recomenda-se a redução manual (Saunders, 2007) e a colocação de sutura em bolsa de tabaco ao redor do ânus. O tratamento cirúrgico deve ser realizado quando não há redução ou quando está gravemente traumatizado e o mesmo consiste na exérese da massa prolapsada (Hedlund, 2008). O prognóstico é favorável mesmo levando em considerações a ocorrência de algumas complicações.

Objetivou-se relatar uma técnica cirúrgica realizada em um filhote felino doméstico como tratamento definitivo de prolapso retal completo.

\section{Relato de Caso}

Um felino, macho, sem raça definida, pesando $0,7 \mathrm{~kg}$ e com dois meses de idade foi atendido no Hospital Veterinário Metropolitano (HVM), Caucaia, Ceará, Brasil. Durante a anamnese foi relatado perda de apetite, hipodipsia, aumento de volume abdominal e presença persistente de massa tecidual que se projetava do ânus do animal. No histórico o tutor revelou ter observado presença de helmintos nas fezes dias antes. O exame físico revelou membranas mucosas discretamente hipocoradas, tempo de preenchimento capilar de 2 segundos, frequência cardíaca com 182 batimentos por minuto, respiratória 18 movimentos por minuto e linfonodos normais para o padrão da espécie. A ausculta cardíaca e pulmonar permaneceu inalterada, pulso e temperatura normais, ausência de ectoparasitas. A massa prolapsada se apresentava com odor pútrido e possuía área necrótica (Figura 1). Solicitou-se perfil hematológico, enzima alanina aminotransferase (ALT), creatinina e ureia como exames complementares.

\section{Resultados}

$\mathrm{O}$ resultado do exame hematológico apresentou leucocitose (27 $\left.\mathrm{mil} / \mathrm{mm}^{3}\right)$ com neutrofilia $(26.300$ células $/ \mu \mathrm{L})$, trombocitopenia (174 $\left.\mathrm{mil} / \mathrm{mm}^{3}\right)$ e discreta anemia $(4,5$ milhões $/ \mathrm{mm}^{3}$ ). A dosagem de ALT mostrou-se acima do normal (233 U/L). No sucessivo, os demais parâmetros encontravam-se dentro das faixas de normalidade para a espécie. 


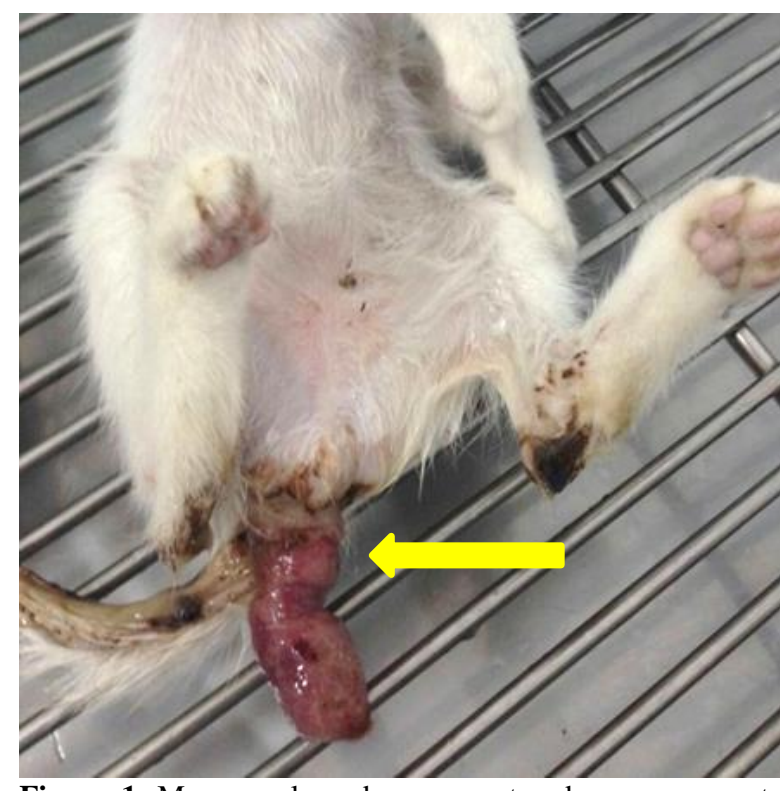

Figura 1. Massa prolapsada com pontos de necrose - seta amarela (Fonte: arquivo pessoal).

$\mathrm{Na}$ abordagem inicial realizou-se a irrigação da área afetada com solução fisiológica, aplicação de gelo e solução açucarada na tentativa de reduzir o edema. Após o insucesso do tratamento conservativo e a determinação da inviabilidade do tecido, o paciente foi encaminhado para o centro cirúrgico.

Instalou-se fluidoterapia com solução ringer com lactato e protocolo pré-anestésico com acepromazina $0,1 \mathrm{mg} / \mathrm{kg}$ e cloridrato de tramadol $2 \mathrm{mg} / \mathrm{kg}$, ambos por via intramuscular. A indução foi feita com proporfol $4 \mathrm{mg} / \mathrm{kg}$ por via intravenosa para posterior intubação e manutenção anestésica com isofluorano.

O procedimento cirúrgico foi realizado através da ressecção da massa e confecção de anastomose removendo o tecido necrosado e desvitalizado. A técnica utilizada para realização da anastomose consistiu em duas ancoragens com o uso de duas agulhas hipodérmicas estéreis tamanho $22 \mathrm{G}$ x 1 " $(25 \mathrm{~mm} \times 0.7 \mathrm{~mm})$ aplicadas transversalmente formando uma "cruz". Em seguida, foram passados fios de Polidioxanona 3-0, por dentro das agulhas para posterior remoção destas e manutenção das ancoragens apenas com os fios de sutura em quatro pontos equidistantes (Figura 2; A). Após isso, aplicou-se suturas em ponto simples separado no padrão de Pauchet. Suturas adicionais foram colocadas para o acabamento da anastomose, no padrão simples separado transfixante total na mucosa, submucosa e seromuscular (Figura 2; B). Ao finalizar a técnica fez-se uma limpeza local com soro fisiológico (Figura 2; C).
Prescreveu-se no pós-operatório a administração de nimesulida $5 \mathrm{mg} / \mathrm{kg}$ s.i.d., amoxicilina $15 \mathrm{mg} / \mathrm{kg}$ b.i.d. e metronidazol 25 $\mathrm{mg} / \mathrm{kg}$ b.i.d., por via oral, durante três dias. Recomendou-se, também, o uso de clorexidina $1 \%$ e medicação tópica a fim de auxiliar na higiene local da ferida cirúrgica. Ademais, indicaram-se dietas de baixo resíduo, uso de probióticos e caminhadas pós-alimentação para facilitar evacuação e prevenir a recorrência do quadro.

Recomendou-se, também, o uso de clorexidina $1 \%$ e medicação tópica a fim de auxiliar na higiene local da ferida cirúrgica. Ademais, indicaram-se dietas de baixo resíduo, uso de probióticos e caminhadas pós-alimentação para facilitar evacuação e prevenir a recorrência do quadro. Passados dez dias o paciente retornou para avaliação. Não foram observados sinais de infecção e a anastomose permaneceu estável. O animal ingeria alimento e possuía trânsito intestinal normal.

\section{Discussão}

A patologia apresentada neste relato ocorreu em um indivíduo de pouco mais de um mês de idade. Esse dado confirma a ocorrência mais frequente dessa patologia em animais com esse perfil epidemiológico, conforme verificado por Shiju et al. (2016). Além disso, Wachask (2002) também observou uma maior incidência dessa patologia em indivíduos abaixo de um ano de idade, podendo estar associado a intussuscepções, enterites e endoparasitismo. Corroborando esses autores o paciente em questão apresentava um quadro de helmintose, o que poderia ter levado a um tenesmo prolongado e a justificava da ocorrência do prolapso retal acontecer em virtude de uma condição clínica secundária.

$\mathrm{O}$ quadro de prolapso retal do felino em questão demonstrava regiões de necrose e infecção, levando a decisão de tratamento cirúrgico com ressecção retal da massa. Segundo Gaston (1951), a técnica pode resultar em complicações caso haja lesão de alguns nervos, resultando em algumas complicações, tais como: incontinência fecal e outros distúrbios de defecação. Tais complicações foram observadas no paciente durante a primeira semana posterior a cirurgia. O mesmo apresentava aumento da frequência de defecação e teor líquido das fezes. Isso pode ocorrer quando há dano ou ruptura das fibras sensitivas aferentes ao redor do reto. Nesse quesito, Landon et al. (2007) afirmaram que é 
possível o retorno da defecação normal uma vez que o esfíncter anal externo é deixado intacto. Passadas algumas semanas não foi observado incontinência fecal no felino, mesmo que esta seja uma complicação relativamente frequente nesses tipos de pacientes.

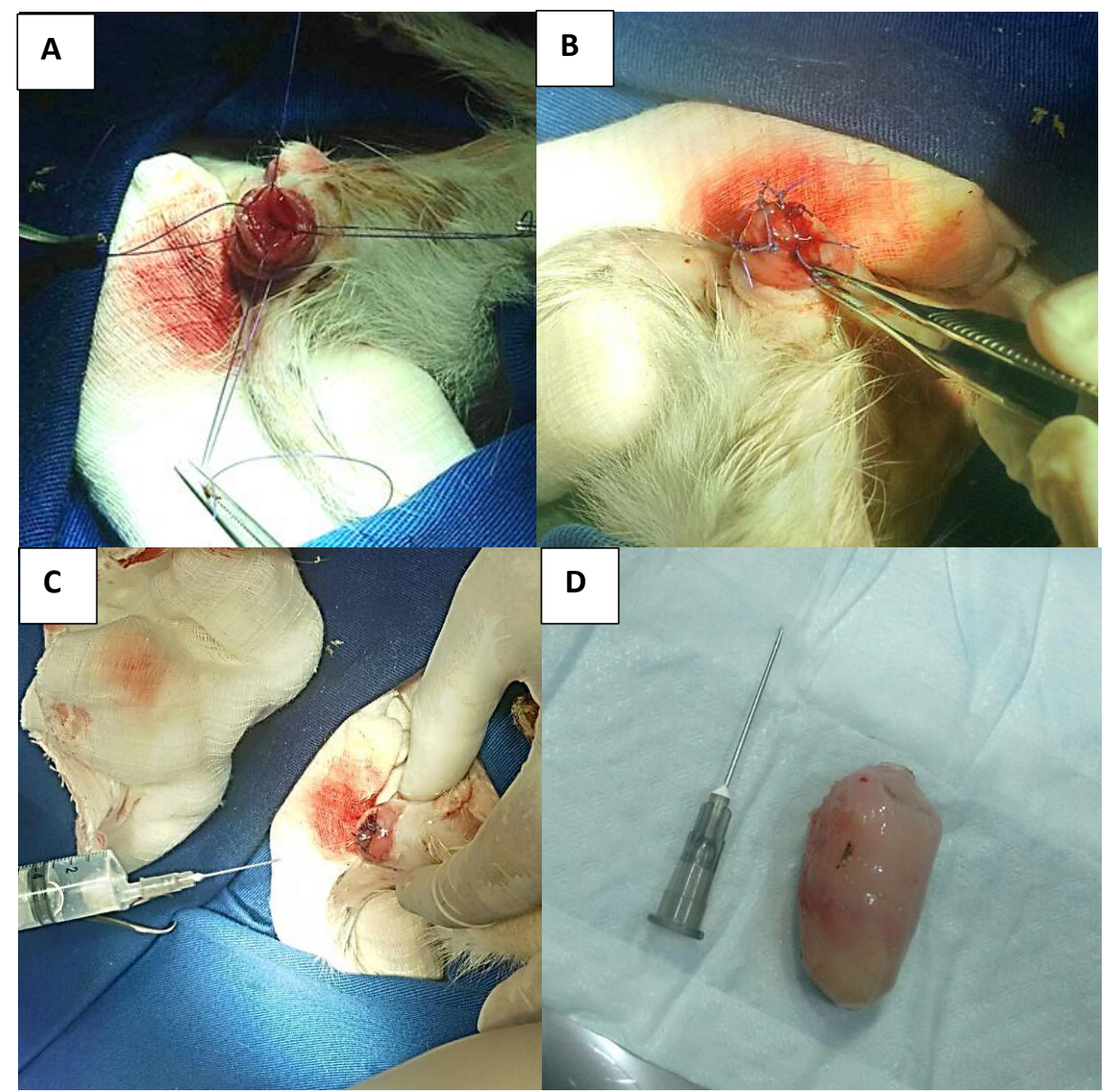

Figura 2. Ressecção de prolapso retal de felino. A- Ancoragem com fio de Polidioxanona 3-0 em padrão de transeção total em quatro pontos equidistantes. B- Suturas para realização de anastomose. C- Limpeza local com soro fisiológico. D- Massa removida desvitalizada com aproximadamente $3,5 \mathrm{~cm}$.

Uma vez que o reto se mostrava muito afetado e com sinais de putrefação, optou-se por realizar a amputação da massa e não a colompexia como demonstrado por Morello et al. (2008). Ademais, neste caso, a escolha dessa técnica baseou-se no estado deteriorado em que se encontrava o animal. De acordo com Saunders (2007), em alguns casos, é possível reduzir o prolapso através da sutura de contenção. Neste estudo, o tratamento do prolapso foi feito através da ressecção da porção inviável.

A incontinência pode resultar da ruptura das fibras aferentes localizadas dentro ou à volta do reto. Sendo assim, na maioria das cirurgias retais é improvável que o nervo pudendo interno e caudal retal sejam danificados. Da mesma forma, mesmo com a extensão do reto, o esfíncter anal externo é deixado intacto.

\section{Conclusões}

O prolapso retal pode desenvolver complicações como o edema e a necrose tecidual tornando impossível a redução manual como tratamento conservador. Neste relato, a ressecção retal demonstrou-se uma alternativa viável na abordagem de prolapso retal em felinos em quadros complicados.

\section{Referências Bibliográficas}

Cunha, M. G. M. C., Pelizarri, C., Seraffin, G., Cunha, J. P. M. C. M., Sampaio, K. O., Sousa Filho, R. P. \& Pippi, N. L. 2015. Rectal prolapse secondary to vesicourachal diverticula in a cat. Ciência Animal, 25, 35-39.

Gaston, E. A. 1951. Physiological basis for preservation of fecal continence after resection 
of rectum. Journal of the American Medical Association, 146, 1486-1489.

Hedlund, C. S. 2008. Cirurgia dos sistemas reprodutivo e genital. In: Fossum, T. W. (ed.) Cirurgia de pequenos animais. Elsevier, Rio de Janeiro.

Landon, B. P., Abraham, L. A., Charles, J. A. \& Edwards, G. A. 2007. Recurrent rectal prolapse caused by colonic duplication in a dog. Australian Veterinary Journal, 85, 381-385.

Morello, E., Martano, M., Squassino, C., Iussich, S., Caccamo, R., Sammartano, F., Zabarino, S., Bellino, C., Pisani, G. \& Buracco, P. 2008. Transanal pull-through rectal amputation for treatment of colorectal carcinoma in 11 dogs. Veterinary Surgery, 37, 420-426.

Niebauer, G. 1993. Técnicas atuais em cirurgia de pequenos animais. In: Bojrab, M. J. (ed.) Current Techniques in Small Animal Surgery. Lea and Febiger, Philedelphia.

Raut, B. M., Raghuwanshi, D. S., Upadhye, S. V., Gahlod, B. M., Khante, G. S. \& Borghare, A. P. 2008. Hormonal management of rectal prolapse in a turtle. Veterinary World, 1, 248.
Saunders, W. B. 2007. Kirk's Current Veterinary Therapy, Philadelphia.

Shiju, M. S., Enbavelan, P. A., Gupta, C., Prabhavathi, H., Ramprabhu, R., Pazhanivel, N. \& Prathaban, S. 2016. Rectal prolapse associated with intussusception and prophylactic colopexy in dogs-A Review of Five Cases. Indian Veterinary Journal, 93, 7476.

Wachask, M. J. 2002. Rectal and anal prolapse. In: Tilley, L. P. \& Smith Júnior, F. W. K. (eds.) The 5-minute veterinary consult. Lippincott Williams \& Wilkins, Baltimore, Maryland, USA.

Article History:

Received 17 September 2017

Accepted 12 October 2017

Available online 22 February 2018

License information: This is an open-access article distributed under the terms of the Creative Commons Attribution License 4.0, which permits unrestricted use, distribution, and reproduction in any medium, provided the original work is properly cited. 